\title{
The Gretchen question in autosomal-dominant polycystic kidney disease research
}

\author{
Friedrich C. Luft
}

Published online: 8 February 2011

(C) Springer-Verlag 2011

Autosomal-dominant polycystic kidney disease (ADPKD) affects about 1 of every 1,000 persons on earth. Half of affected persons can expect to develop end-stage chronic renal disease (ESRD) during their lifetimes. The condition represents $15 \%$ of dialysis populations worldwide. Other affected persons will die prematurely from various cardiovascular causes. The cloning of PKD1 and PKD2 spurred an enormous amount of research into ADPKD. PKD1 encodes polycystin-1, a large transmembrane protein that interacts with polycystin-2, a nonselective calcium channel that localizes to primary cilia in the renal epithelium. PKD2 encodes polycystin-2. Mutations in these two genes make up the vast majority of ADPKD patients. Discovery of these proteins as present in cilia placed ADPKD into the category of the human "ciliopathies", which includes a host of other complex genetic diseases, notably the nephronophtheses, the Bardet-Biedl syndromes, and a dozen other conditions, many of which also feature renal cysts [1]. The cilia proteins responsible for these conditions participate in Wnt signaling, Hedgehog signaling, platelet-derived growth factor (PDGF) signaling, fibroblast growth factor (FGF) signaling, and interact with the mammalian target of rapamicin (mTOR) signaling pathway (Fig. 1). The ciliary location of PC-1 and PC-2 and the presence of cilia on the luminal surface of renal tubular cells are consistent with the idea that cilia are important for the maintenance of tubular planar cell polarity [2]. Animal models suggested that mTOR inhibitors could ameliorate cyst growth perhaps by assuming the role that mutated

F. C. Luft $(\bowtie)$

Experimental and Clinical Research Center,

Max-Delbrück Center and Charité Medical Faculty,

Lindenbergerweg 80,

13125, Berlin, Germany

e-mail: luft@charite.de
PC-1 or PC-2 could not fulfill; however, recent clinical trials were disappointing [3].

The cells lining renal tubules generally divide parallel to the tubular axis, which lengthens the tubule rather than increasing tubular diameter. A loss of planar cell polarity could be a precursor to cyst formation. Cysts can occur anywhere along the nephron and destroy the kidney. Only a thousand or so cysts are necessary to eventually destroy the kidney, arising from the $1,000,000$ or so nephrons in each kidney. The autosomal-dominant inheritance and the fact that the disease affects aging adults rather than children, is consistent with the notion that the second allele is somehow "hit" and rendered nonfunctional.

In German, the word "Gretchenfrage" (literally "Gretchen question") refers to a question aiming at the "core of the issue". Inherent in the Gretchen question is the fact that those supplying the answers make a confession, or at any rate a "difficult decision", to obtain the answer. The core of this issue is, "what is the mechanism of cyst growth?" Cyst growth, as we have learned, destroys the remaining renal parenchyma. The existence of elegant conditional and controllable gene-deleted mouse models has provided additional information.

Repair of acute kidney injury is impaired in mice lacking Pkd1 or Pkd2. The kidneys acquire more dilated tubules than wild-type mice. The knockout in adult mice subjected to acute kidney injury exhibits a sensitivity that is similar to changes observed in juvenile mice. These data suggest that the cystic signal may not necessarily require a "second hit" to become manifest. In addition, acute kidney injury accelerates cyst formation. Conceivably, cyst formation could depend upon the occurrence of either a somatic mutagenesis or an injury event. Either of these events could be construed as a "second hit" that interacts with heterozygosity at one of the ADPKD loci to cause the 
Fig. 1 The $\mathrm{N}$ and C-terminal components of the $\mathrm{PC} 1$ protein can be cleaved. The extracellular domain, however, remains covalently attached to the molecule. The C-terminal tail fragment is able to translocate to the nucleus with components of the Wnt pathway, STAT6/p100, and perhaps other regulators of transcription. The C-terminal tail cleavage is stimulated by the presence of PC2. The interaction is necessary for PC2 to function as a calcium channel. Furthermore, multiple direct and indirect interactions allow the polycystin proteins to inhibit or stimulate pathways involved in cell growth and differentiation (adapted from [2])
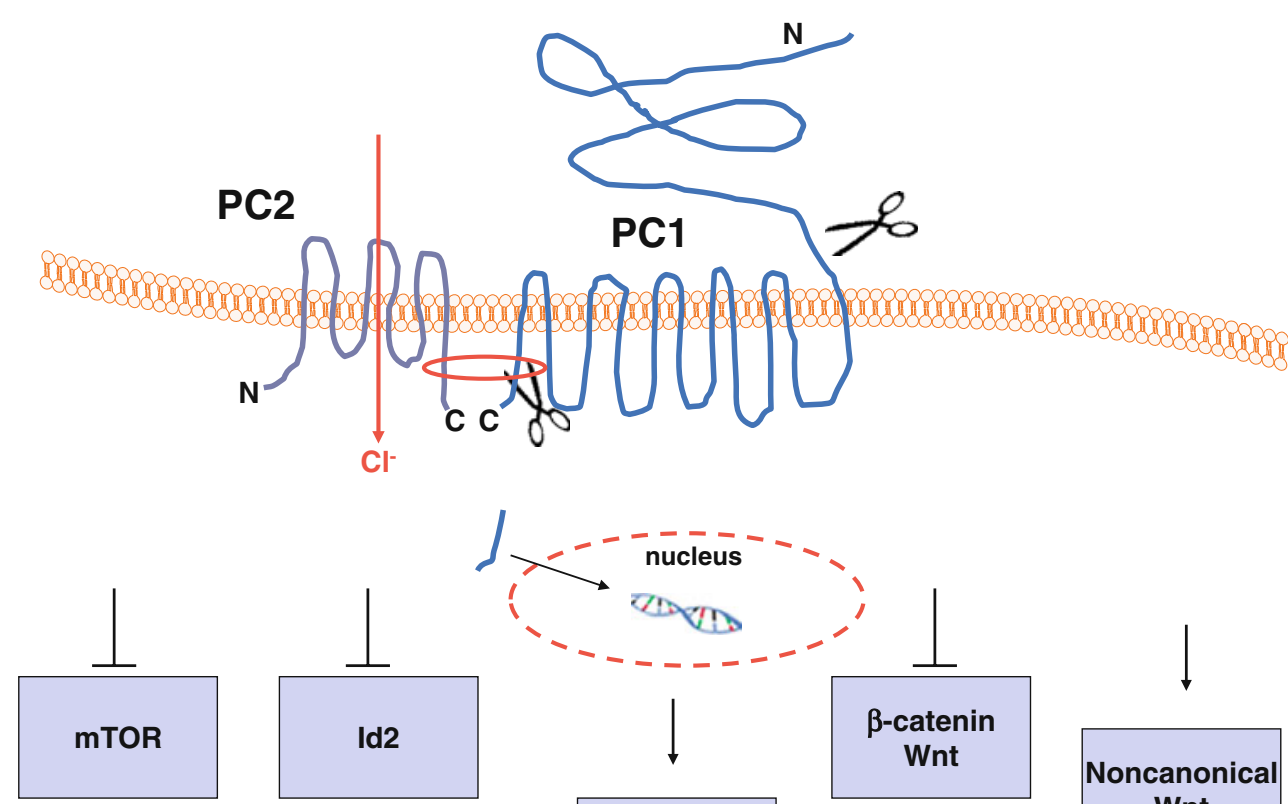

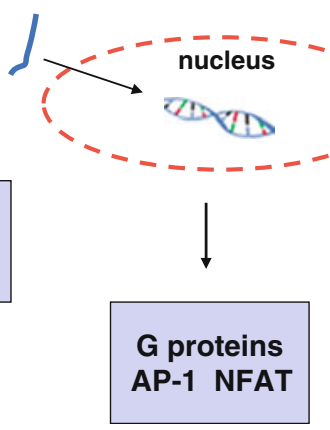

Belibi et al. [3] harvested mural cells from renal cysts derived from patients with ADPKD. They prepared the cells in culture and measured cAMP responses to agonists, transepithelial anion currents, and extracellular signalrelated kinase expression. Belibi et al. [3] were able to show that endogenous adenylyl cyclase agonists promote cell proliferation and electrolyte secretion of human ADPKD and ARPKD cells in vitro. Their evidence further suggested that the cyst-derived cells had their origin in the collecting duct, rather than other tubular sites. The renal collecting duct system possesses two major cell types. The principal cells mediate the collecting duct's influence on sodium and potassium balance via sodium and potassium channels located on the cell's basolateral membrane.

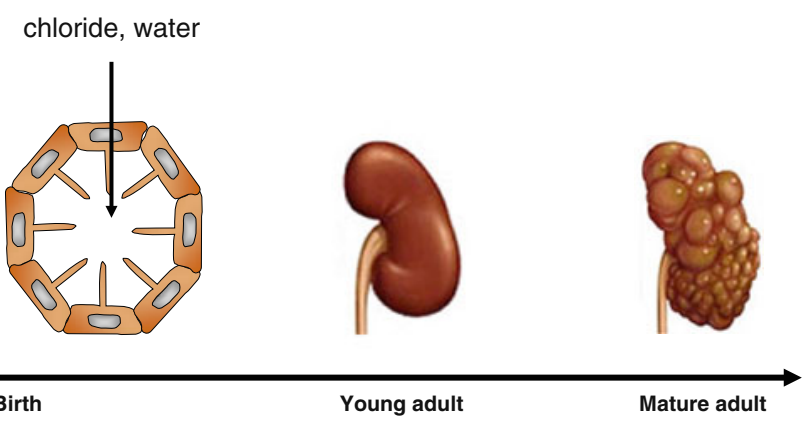

Fig. 2 Defects on PC1 or PC2 within the cilia leads to aberrant gene transcription, cell proliferation, and altered ion secretion that leads to fluid-filled cysts. As the cysts balloon from individual nephrons, the renal parenchyma is displaced and eventually destroyed. The cysts appear to be commonly derived from collecting duct epithelium, probably principal cells. The process requires many years, making interventions possible (adapted from [2]) 


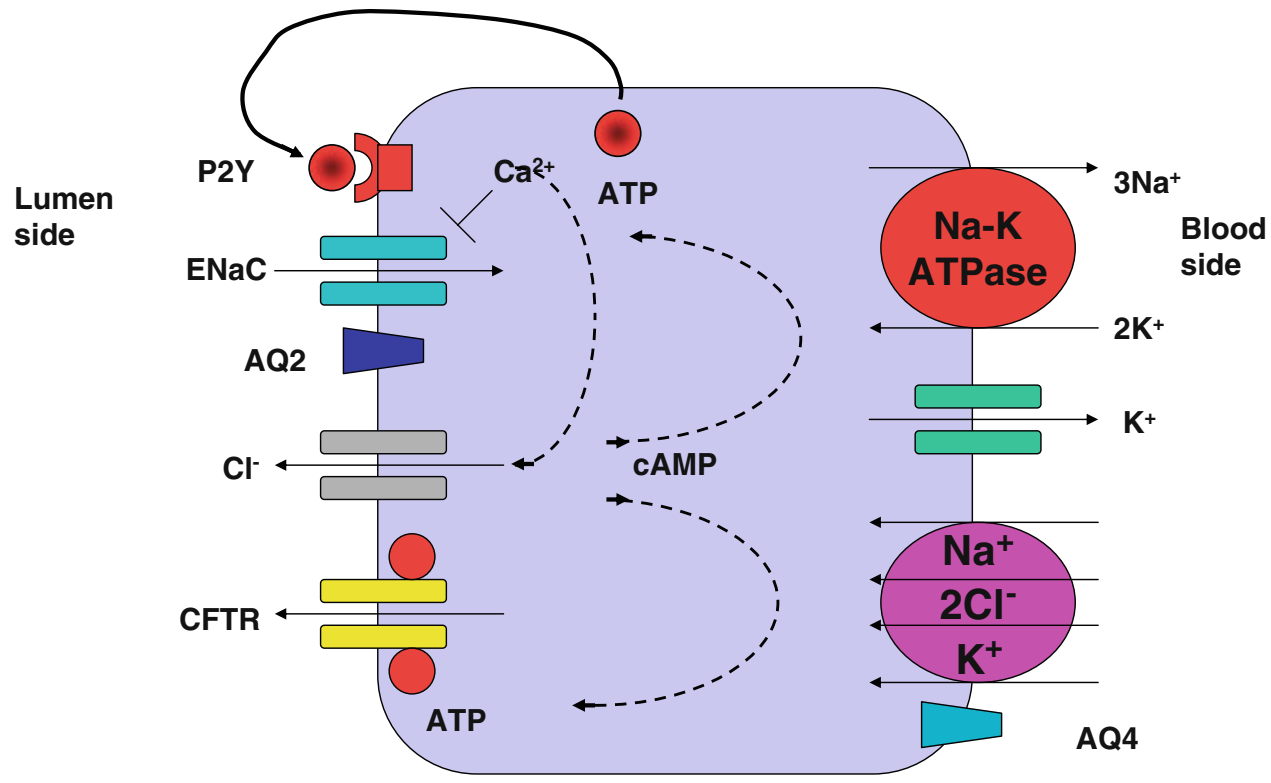

Fig. 3 The authors speculate on an increase in ATP that autosignals via $\mathrm{P} 2 \mathrm{Y}$ receptors to decrease $\mathrm{ENaC}$ activity and increase $\mathrm{Cl}$ channel activity. An increase in adenylate cyclase could also increase ATP to augment CFTR. Also shown is AQ2 present in principal cells,

Furthermore, vasopressin via V2 receptors determines the expression of aquaporin channels on the cell surface. Vasopressin allows the principal cell to control the quantity of water, which is reabsorbed. Vasopressin is a major adenylate cyclase agonist in principle cells and cAMP promotes cystogenesis. These findings and a host of similar data have led the use of vasopressin (V2) antagonists to retard cyst growth in ADPKD. The intercalated cells of the collecting duct come in $\alpha$ and $\beta$ varieties and primarily participate in acid-base homeostasis.

A commonly employed cell culture model of ADPKD employs the Madin Darby Canine Kidney (MDCK) cell line. Mangoo-Karim et al. [6] showed years ago that MDCK cells, cultured in hydrated-collagen gel, produced polarized monolayered epithelial cysts when intracellular cAMP was increased by prostaglandin E1, vasopressin, cholera toxin, forskolin, or 8-bromoadenosine 3',5'-cyclic monophosphate. They also observed that 3-isobutyl-1methylxanthine, a nucleotide phosphodiesterase inhibitor, potentiated all agonists. Furthermore, Mangoo-Karim et al. showed that the cell proliferation component of cyst enlargement was accelerated by cAMP agonists. Chloride levels were higher in the cyst fluid and the secreted fluid than in the bathing medium. Mangoo-Karim et al. concluded that the development of MDCK cysts was dependent on cAMP.

In this issue, Buchholz et al. [7] pursued the importance of collecting duct cells in cyst formation further. They studied MDCK cell subclones. The C7 subclone resembles principal cells, while the $\mathrm{C} 11$ subclone resembles interca- although the water movement would follow solute transport directions in this model (modified from [5]). Cyst cells are not principal cells, although likely derived from principle cells

lated cells. The authors found that cultured cysts developed only from C7-derived clones and not from C11-derived clones. Their findings imply that principal cells are indeed the source of renal cysts. In vitro cyst growth in their model occurred upon elevation of intracellular cAMP and mainly driven by fluid secretion, rather than increased cell proliferation. The cAMP-dependent fluid secretion was found to depend on extracellular ATP and to act synergistically with purinergic signaling, since the ATP scavenger apyrase and the $\mathrm{P} 2 \mathrm{Y}$ receptor inhibitor suramin-reduced cAMP-driven fluid secretion, while increasing extracellular ATP potentiated cAMP-mediated cyst growth. These findings are in accord with the earlier report by Turner et al. [6], who showed that stimulation of endogenous $\mathrm{P} 2 \mathrm{Y}$ receptors by extracellular ATP increases growth of MDCK cysts via cAMP-dependent activation of the ERK pathway. They suggested that P2Y receptor antagonists could have therapeutic potential in reducing cyst size and slowing disease progression.

Cystic cells are markedly different from normal collecting duct principal cells, and surely also from MDCK cells. Chloride presumably enters from a basolateral $\mathrm{Na}^{+}-2 \mathrm{Cl}^{-}-\mathrm{K}^{+}$ transporter driven by the gradient supplied by $\mathrm{Na}^{+}-\mathrm{K}^{+}-$ ATPase (Fig. 3). The chloride probably mostly exists via the CFTR. Presumably, the chloride transport into the cyst lumen drives sodium and water secretion down transmembrane potential and osmotic gradients. The location of $\mathrm{P} 2 \mathrm{Y}$ receptors and the epithelial sodium channel $\mathrm{ENaC}$, as well as aquaporin-2 is correct for principal cells; however, for actual cyst cells, I am less certain. Cysts can apparently 
maintain tight junctions, or their maintenance could not be maintained.

Novel therapies to diminish the growth of cysts, with even less-than dramatic effects, could have a substantial effect on clinical outcomes. Such studies are currently being performed [7]. Two mTOR inhibitor trials have been completed that gave less than the expected result [8]. There are numerous explanations, not the least of which is that treatment is started when the disease is relatively faradvanced, albeit still asymptomatic. V2 receptor antagonist studies are underway. Also in the works are studies involving long-acting somatostatin analogs. Various antihypertensive trials and even sympathectomy is being considered. To my knowledge, no trials of P2Y inhibitors are as yet underway. My interpretation would be that the therapists address the "difficult decision" regarding the Gretchen question. Only direct testing will supply an answer. Suffice it to say, the intense research is surely worth it and help is underway for these patients.

Respectfully, Friedrich C. Luft

\section{References}

1. Cardenas-Rodriguez M, Badano JL (2009) Ciliary biology: understanding the cellular and genetic basis of human ciliopathies. Am J Med Genet C Semin Med Genet 151C:263-280

2. Chapin HC, Caplan MJ (2010) The cell biology of polycystic kidney disease. J Cell Biol 191:701-710

3. Belibi FA, Reif G, Wallace DP, Yamaguchi T, Olsen L, Li H, Helmkamp GM, Grantham JJ (2004) Cyclic AMP promotes growth and secretion in human polycystic kidney epithelial cells. Kidney Int 66:964-973

4. Mangoo-Karim R, Uchic M, Lechene C, Grantham JJ (1989) Renal epithelial cyst formation and enlargement in vitro: dependence on cAMP. Proc Natl Acad Sci USA 86:6007-6011

5. Buchholz B, Teschemacher B, Schley G, Schillers H, Eckardt K-U (2011) Formation of cysts by principal-like MDCK cells depends on the syndergy of camp and ATP-mediated fluid secretion. J Mol Med. doi:10.1007/s00109-010-0715-1

6. Turner CM, King BF, Srai KS, Unwin RJ (2007) Antagonism of endogenous putative $\mathrm{P} 2 \mathrm{Y}$ receptors reduces the growth of MDCKderived cysts cultured in vitro. Am J Physiol Renal Physiol 292: F15-F25

7. Torres VE, Harris PC (2009) Autosomal dominant polycystic kidney disease: the last 3 years. Kidney Int 76:149-168

8. Watnick T, Germino GG (2010) mTOR inhibitors in polycystic kidney disease. N Engl J Med 363:879-881 